\title{
Electromagnetic Field of Resonance Linear Motion Actuator and its Finite Element Analysis which Applied to Coal Mine
}

\author{
Xianyi Qian ${ }^{\text {a,*, }}$ Yiming $\mathrm{He}^{\mathrm{a}}$ \\ ${ }^{a}$ School of Electronic Information \& Electric Engineering, Changzhou Institute of Technology, CZU, \\ Changzhou, China
}

\begin{abstract}
In this paper, we have introduced the structure of resonance linear motion actuator which applied to coal mine. And we have built two-dimensional analyzing model by using finite element method. On the basis of the analysis, we have calculated the motor's air-gap field and magnetism, and we also have studied the affection of motor which caused by permanent magnet's size. We have studied motor's winding current when it operates at constant amplitude, and the corresponding experiment results have verified the correctness of finite element model. We have studied the numerical value calculation of resonance linear motion actuator and calculation of its magnetism characteristic. What we have analyzed shows that the air-gap field of motor changes continuously and the magnetism is proportional to winding current. And the results can help technologists to design this kind of motor.
\end{abstract}

Index Terms: Resonance linear motion actuator; Structure model; Finite element analysis; Numerical calculation; Characteristics of suction

(C) 2011 Published by MECS Publisher. Selection and/or peer review under responsibility of the Research Association of Modern Education and Computer Science.

\section{Introduction}

In mine production, most of the accidents are caused by motor failure. And the death toll accounts for $40 \%$ in all mine accidents. In this paper, we have analyzed the conveyance secure management of DC motor which used widely. How to improve its reliability is the main study purpose here.

The technical and economical specifications have relations with devices of motor's structure. In recent years, because of the development of computer CAD technology, the numerical calculation of electromagnetic structure is more and more important. Because the pure calculation of magnetic path can't reflect the field intensity distribution of magnetic structure exactly. We can calculate the electromagnetic force's characteristic

* Corresponding author.

E-mail address: hbxfqxyqxy_123@163.com 
exactly by means of analysis of field. [1]

The essence of evaluate the boundary value of magnetic field is that solving the Poisson Equation or Laplace Equation which can meet the boundary conditions. Because the interface between the boundary of motor's magnetic system and its inner medium is very complex and ferromagnetic medium is also included in the solving region, which make constant magnetic differential equation have the nonlinear characteristics. So, it is hard to solve directly, and we can only calculate the approximate solution by means of numerical method. Finite element method has been applied to solve numerical analysis of magnetic field widely in many numerical calculation systems. Finite element method is the method which solves problem by means of variation principle and discrete solutions. The electromagnetic field in motor is the problem of partial differential equation's boundary value, and finite element method doesn't use it as object to solve. It uses the conditional problem of variation as object to solve the problem of electromagnetic field. Here, we first have calculated the distribution of amount of field, and then solved its characteristic of electromagnetic force.

\section{Numerical calculation of linear motion actuator's magnetic field}

The key study of linear motion actuator is the characteristic of absorbing force. And the calculation of absorbing force's characteristic is based on the value of air-gap field. So, we should first calculate the value of magnetic field before analyzing the absorbing force's characteristics.

Linear motion actuator usually uses the two kinds of structures: U-type and E-type. In this paper, we have taken E-type linear motion actuator for example, and we only calculated its half symmetry. Fig. 1 shows it calculation model.

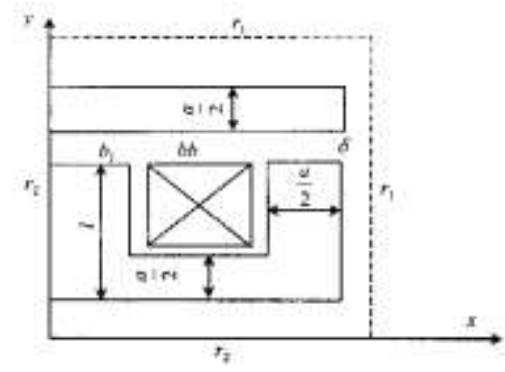

Fig. 1. Boundary of magnetic field

According the calculus of variations, we can get the partial differential equation which is in twodimensional domain:

$$
\sum \frac{\partial}{\partial x}\left(\gamma \frac{\partial A}{\partial x}\right)+\frac{\partial}{\partial y}\left(\gamma \frac{\partial A}{\partial y}\right)=-J
$$

In equation (1), the parameter of $\gamma$ means reluctivity and the parameter of $\mathrm{J}$ means density of electromagnetic winding's current.

$$
\vec{B}=B_{x} i+b_{y} j
$$

In this equation, $B_{x}=\frac{\partial A}{\partial y}, B_{y}=\frac{\partial A}{\partial x}$.

According the chosen E-type linear motion actuator, we have used magnetic field boundary as dirichlet boundary: 
$\Gamma_{1}: A=0$

So the functional extremum problem of condition is equivalent to the boundary value of magnetic field partial differential equation.[2] We must divide field domain into lots of units when we analyze the field domain which shown in Fig.1 by using finite element numerical analysis. And we have written the program which can divide field domain into lots of triangular units automatically.

On the condition of given air-gap and winding's current, we can conclude the linear equation by means of filed domain discretization and finite element numerical analysis.

$[\mathrm{k}][\mathrm{A}]=[\mathrm{p}]$

In this equation, $[\mathrm{k}]$ means coefficient matrix; [A] means column vector of magnetic field; [p] means column vector of right item.

To the nonlinear equation which has been shown in equation (4), we can use Newton-Raphson iterative search technique to solve the discrete point magnetic field of $\mathrm{A}$ in filed domain. We also can solve every unit's magnetic density B.

\section{Numerical value of linear motion actuator magnetic absorbing force's characteristics}

The action force of magnet which caused by magnetic field has formed the inner stress of magnetic filed. It is also the vertical pressure between the strains of magnetic lines of forces. So, the electromagnetic absorbing force can be concluded by calculating the strains on the surfaces of the magnet.

$$
F=\iint P d s
$$

In equation (5), the parameter of $\mathrm{P}$ means the action force on the surface of unit, $\mathrm{N} / \mathrm{m}^{2}$.

$$
P=\frac{1}{\mu_{0}}(n g B) B-\frac{1}{2 \mu_{0}} B^{2} n
$$

In the equation (6), ${ }^{\mu}$ means air magnetic permeability; $\mathrm{F}$ is the electromagnetic absorbing force which acted on magnetizer; $\mathrm{N}$ is the unit vector which along the direction of normal; $\mathrm{B}$ means density of magnetic induction.[3]

Equation (5) is the integral vector, which the sum of absorbing forces on every element by means of integral. Once we have gotten the discrete value of magnetic field, we can calculate electromagnetic absorbing force in accordance with equations (5) and (6) conveniently. We only need a integral surface and the magnet is closed, so the any integral surface $S$ which surrounding this object can get the same absorbing force. If the integral surface of S is in air, we needn't consider the nonlinear probable of magnet. So we can choose easy integral surface. If the surface clings to the surface of magnet, we can think that B is vertical to the surface of magnet because iron's magnetic permeability $\mu$ is bigger than air magnetic permeability $\mu_{0}$. This means that the direction of $B$ is the normal direction $n$ of integral surface $\left((\mathbf{n} \cdot \mathbf{B}) \mathbf{B}=\mathrm{B}^{2} \mathbf{n}\right)$. On the other hand, the integral way $\mathrm{S}$ is always the center of triangular element, which can increase the precision of calculation.

The thickness of linear motion actuator is always uniform. On the conditions of prescribed thickness, the integral of surface in equation (5) can be represented line integral which along air gas. The absorbing force expression of triangular element is:

$$
F=l_{c} \int_{l}\left(P_{x} i+P_{y} j\right) d l
$$

In the equation (7), there existed the following equations:

$$
P_{x}=\frac{1}{\mu_{0}}\left(n_{x} B_{x}+n_{y} B_{y}\right) B_{y}-\frac{B^{2}}{3 \mu_{0}} n_{x}
$$




$$
P_{x}=\frac{1}{\mu_{0}}\left(n_{x} B_{x}+n_{y} B_{y}\right) B_{y}-\frac{B^{2}}{2 \mu_{0}} n_{y}
$$

In these two equations, $1_{c}$ means the thickness of linear motion actuator and 1 means the integral way of absorbing force.

According the numerical calculation of magnetic field, the magnetic value of every element has been calculated. So we can calculate the corresponding magnetic absorbing force of air-gap and the magnetic absorbing force of current.

$$
F=\sum_{i=1}^{n} P_{i} \Delta S_{i}
$$

In equation (8), $\Delta S_{i}$ means the surface area of element $\mathrm{i} ; P_{i}$ means the surface stress of element $\mathrm{i} ; \mathrm{n}$ means the total number of elements which pass integral way.

\section{Analysis of examples}

We can get the better precision by using magnetic field numerical calculation method to calculate electromagnetic absorbing force. The more denseness of magnetic field domain, the higher precision is. Table.1 shows the calculating model's sizes and parameters. Fig. 1 shows the example which has taken the absorbing force characteristic of linear motion actuator E-type magnetic system.[4] The numerical calculation of magnetic field has been used finite element method, and some units are triangular elements. There are 588 elements and 330 nodes, and the number of inner nodes is 264 .

Table 1. The needed model sizes and parameters

\begin{tabular}{|c|c|c|c|c|}
\hline $\begin{array}{c}\text { Width of middle iron core } \\
(\alpha / \mathbf{m m})\end{array}$ & $\begin{array}{c}\text { Height of iron core } \\
(\mathbf{l} / \mathbf{m m})\end{array}$ & $\begin{array}{c}\text { Thickness of } \\
\text { windings }(\mathbf{b} / \mathbf{m m})\end{array}$ & $\begin{array}{c}\text { Current of } \\
\text { windings (I/A) }\end{array}$ & Number of turns \\
\hline 100 & 140 & 47 & 3.20 & 1104 \\
\hline
\end{tabular}

Fig. 2 shows the magnetic field distribution which from calculation. It shows that there is a little magnetic leakage between air-gap top and two iron core. The electromagnetic force depends on the air-gap density. The little magnetic leakage, the bigger absorbing value is. We can calculate magnetic absorbing force on the basis of magnetic field calculation. So we can solve the absorbing value in accordance with the prescribed air-gap length.[5]

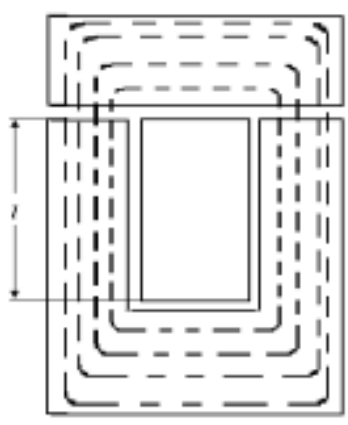

Fig. 2. Distribution of magnetic field (Only the right half) 
Fig.3 shows the comparing between finite element calculation and magnetic path force calculating method. The calculating results are almost equal when the air-gap is small, and the method which using magnetic path calculation only can replace scatted magnetic leakage with lots of centralized magnetic leakage.

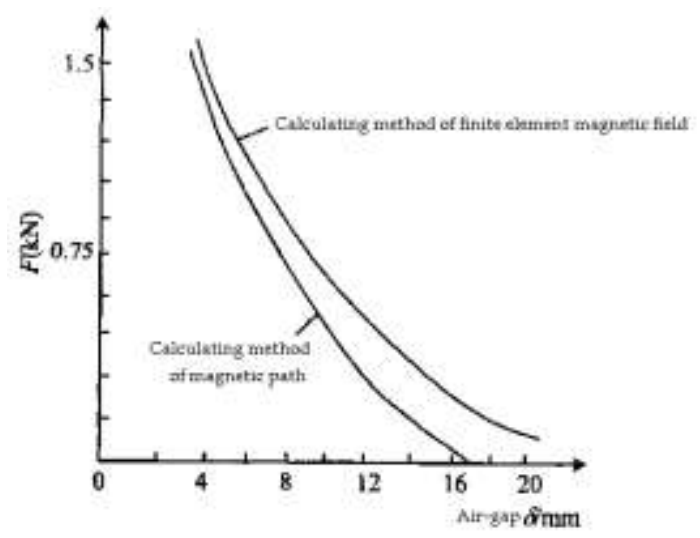

Fig. 3. Characteristic curve of magnetic field's absorbing force

\section{Conclusions}

We have concluded some conclusions by comparing finite element calculating method with calculating force of magnetic pass. There is a little magnetic leakage between air-gap top and two iron core. The electromagnetic force depends on the air-gap density. The little magnetic leakage, the bigger absorbing value is. We can calculate magnetic absorbing force on the basis of magnetic field calculation.

The model of resonance linear motion actuator which used in coal mine is reasonable. And its method about controlling and solving is correct. Because we have considered the affection which caused by magnetic leakage's variation and correctness of motor parameters, the design can meet the requirements of engineering design. The designed model has such characteristics as good reliability, high precision and convenient application. What we have studied provides a kind of new design for the optimization of compressor's system parameters, and provides a effective method which used to design and develop resonance linear motion actuator.

\section{Acknowledgements}

We are very grateful to the Jiangsu Province College Natural Science Foundation of China for the support.

\section{References}

[1] Zhang Jinquan, Chang Yunfeng, Modeling and Simulation of Compressor Driven By Moving-Magnet Linear Motor[J], JOURNAL OF XI'AN JIAOTONG UNIVERSITY, 2007 41(7)

[2] Xia Yongming, Lu Qinfen, Modeling and Analysis of a Novel Linear Oscillatory Motor With Two Divided Stators[J], TRANSACTIONS OF CHINA ELECTROTECHNICAL SOCIETY, 2007 22(12)

[3] Wang Xuping, Static Characteristic Analysis of Several Basic Kinds of Linear Oscillatory Motor[J],MICROMOTORS, 2003 36(3) 
[4] Chen Jiefeng, The Oscillation Characteristic Analysis of a Special Linear Motion Oscillation Motor[J], SMALL \& SPECIAL ELECTRICAL MACHINES, 2002 30(5)

[5] Wang Xinhua, ANALYTICAL CALCULATION OF NO-LOAD AIR-GAP MAGNETIC FIELD AND BACK ELECTROMOTIVE FORCE IN BRUSHLESS DC MOTOR[J], PROCEEDINGS OF THE CHINESE SOCIETY FOR ELECTRICAL ENGINEERING, 2003 23(3) 